\title{
«Ein Arzt kann die Menschen nicht verlassen»
}

\author{
Maja Hessa, Bigna Rambert ${ }^{\text {b }}$ \\ ${ }^{a}$ Dr. med., med. pract., Präsidentin von medico international schweiz, Mitglied der FMH \\ ${ }^{b}$ Dr. med., Fachärztin für Psychiatrie und Psychotherapie, Mitglied der FMH
}

Die Ärztin Maja Hess, Präsidentin der Nichtregierungsorganisation (NGO) medico international schweiz, und Therese Vögeli von der Geschäftsstelle besuchten Anfang Mai Rojava. Maja Hess fuhr auch nach Shehba nördlich von Aleppo. Dorthin flüchtete ein grosser Teil der Bevölkerung aus Afrin, nachdem dieser westlichste Kanton der Demokratischen Föderation Nordsyriens (auch «Rojava» genannt) von der türkischen Armee Mitte März definitiv besetzt worden war. Die kleine Schweizer NGO unterstützte seit 2015 sowohl in Kobanê als auch in Qamishlo zwei kleine Projekte lokaler kurdischer Partnerorganisationen aus dem Gesundheitsbereich. Der erneute Krieg und die Flüchtlinge aus Afrin brachten neue Herausforderungen.

Auszug aus einem Gespräch von Maja Hess mit Dr. Abdokadir Hasin aus der Stadt Afrin:

«Vor dem Krieg* haben wir Patienten behandelt. Es hat uns Ärzten an nichts gefehlt. Als die Angriffe stärker wurden, flüchtete die Bevölkerung aus den umliegenden Dörfern in die Stadt. Es war ja noch mitten im Winter. Ich bin bis zum Schluss in Afrin geblieben und habe im Krankenhaus und in meiner Privatklinik

\section{«Als wir Afrin verliessen, ging es nur noch} darum, zu überleben.»

gearbeitet. Denn ich bin Arzt, und die Arbeit des Arztes ist menschlich, ethisch und sozial. Ein Arzt kann die Menschen nicht verlassen, das wäre, als ob ein Fisch das Meer verlassen könnte. Wenn er das Meer verlässt, stirbt er. Wir sind Teil unserer Gesellschaft, also dienen wir auch der Gesellschaft. Deshalb habe ich Afrin erst verlassen, als die Angriffe sehr heftig wurden und dabei auch das Krankenhaus zerstörten. Vor dem Krieg sind dorthin täglich hunderte von Menschen zur Behandlung gekommen, weil es gute Ärztinnen und Ärzte gab und die nötigen Geräte vorhanden waren. Unter anderem verfügte das Spital über eine sehr gute Kinderstation und eine grosse Frauenabteilung. Es war ein Krankenhaus für alle, das erste dieser Art in Rojava.»

«Die türkische Armee zerbombte das Krankenhaus, bis es nicht mehr nutzbar war. Das Personal war komplett schockiert und sah sich grösstenteils nicht mehr imstande zu arbeiten. Leider hatten wir keine Möglichkeit und keine Zeit, die technischen Geräte zu retten. Als wir Afrin verliessen, ging es nur noch darum, zu überleben. Sogar auf der Flucht wurden die Menschen von Bomben getroffen. Wir konnten die Toten nicht einmal bergen. Einige Flüchtende überfuhren mit ihren Fahrzeugen sogar die Leichen, weil sie solche Angst hatten. So weit ist es in dieser schlimmen Gewaltsituation mit der Menschlichkeit gekommen. Die Menschen waren vollkommen ausser sich, hatten vor Angst den Verstand verloren. In anderen Staaten werden sogar kleine Tiere gerettet, wenn sie sich ein Bein gebrochen haben. Aber wenn hier Menschen mit Bomben und Luftangriffen umgebracht werden, interessiert dies niemanden. Ist das nicht eine sonderbare Welt? Das ist keine Menschlichkeit, das ist nur Brutalität und Barbarei. Es ist kaum zu beschreiben. Wir haben sehr Unethisches und Unmenschliches erlebt. Die Menschen in Afrin waren auf so etwas nicht vorbereitet.»

«Wir arbeiten mit den einfachsten Mitteln. Unsere Diagnosen stellen wir grösstenteils klinisch.»

«Hier in Shehba bauen wir wieder eine Gesundheitsversorgung auf. Das Gesundheitsteam hat jetzt 37 Mitglieder: 7 ÄrztInnen, PflegerInnen und andere Mitarbeitende. Wir eröffnen hier sogar ein kleines 


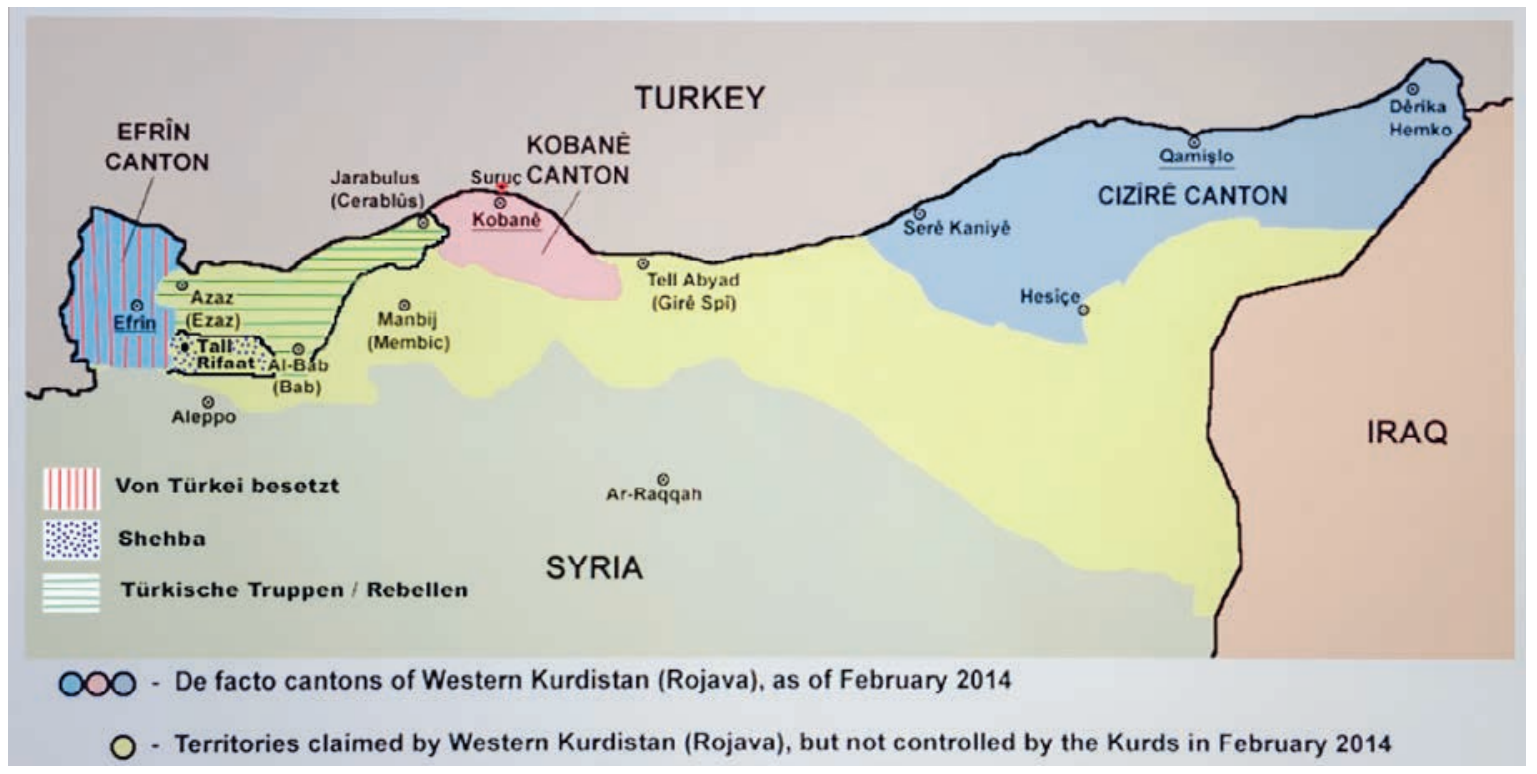

Der Kartenausschnitt der Region vermittelt einen Eindruck von der aktuellen Situation (Aktualisierung Juli 2018 durch die Autorinnen).

Krankenhaus. Wir arbeiten mit den einfachsten Mitteln. Unsere Diagnosen stellen wir grösstenteils klinisch. Besonders verbreitet sind Bronchitis, Herzkrankheiten, Tuberkulose, Leishmaniose, Windpocken, Hepatitis B und C. Wir sind eingekesselt, und das syrische Regime lässt die wenigsten Patientinnen nach Aleppo zur Behandlung durch. Die Flüchtlinge aus Afrin leben hier auf engstem Raum. Oft 20 Personen zusammen in einem Zimmer oder im Zelt im Flüchtlingscamp. Materielle Unterstützung wurde uns aus anderen Teilen Rojavas geschickt, aus Qamishlo und Heseke. Der Kurdische Rote Halbmond ist sehr engagiert, um die Gesundheitsversorgung zu verbessern. Auch der Syrische Rote Halbmond hat uns ein wenig geholfen.»

Fragen von Bigna Rambert an Maja Hess (Präsidentin) und Therese Vögeli (Geschäftsleitung) von medico international schweiz:

Anfang Mai habt ihr die autonome Kurdenregion in Nordsyrien besucht. Wie kam diese Reise zustande? Maja Hess: Wir wurden von der unabhängigen Stiftung der Freien Frau in Rojava (WJAR) und der Solidaritätsplattform «Women rise up for Afrin» eingeladen. Im Vorfeld hatte die kurdische Diplomatie für uns die Realisierung der Reise in die Region Shehba mit den diversen politischen und militärischen Instanzen ausgehandelt. Kurz vor unserem Ziel durchquerten wir die völlig zerbombte, aber bereits mit neuem Leben gefüllte Stadt Aleppo. Auf der kurzen Fahrt von Aleppo nach Shehba wurden wir gebeten, ein Kopftuch umzu- binden, um nicht sofort als Fremde wahrgenommen zu werden. Wir waren die erste internationale Delegation, eine Frauendelegation übrigens, der es gelungen ist, bis nach Shehba zu gelangen.

Dr. Hasin harrte bis zuletzt in seiner Stadt Afrin aus, um für die Zivilbevölkerung einen ärztlichen Minimalstandard aufrechtzuerhalten. Viele kurdische Ärztinnen und Ärzte fühlen sich ihrem Beruf, aber auch ihrem Volk verpflichtet und riskieren angesichts der vielen immer wieder aufflammenden Kriegsherde auch ihr eigenes Leben. Medico international schweiz entschloss sich im Januar 2015 für einen neuen

Projektschwerpunkt in den Kurdengebieten. Warum? Maja Hess: Seit der Gründung von medico international schweiz 1937, damals noch unter dem Namen Centrale Sanitaire Suisse (CSS), hat sich diese Organisation nie als neutral bezeichnet, sondern diejenigen unterstützt, welche sich gegen Unrecht auflehnten, damals gegen den Faschismus. In diesem Sinne helfen wir seit 2015 dem kurdischen Selbstverwaltungsprojekt Rojava in Nordsyrien mit kleinen punktuellen Projekten beim Wiederaufbau und Aufbau ihrer Gesundheitsstrukturen. Als kleine NGO haben wir in anderen Ländern vor allem mit medizinischen Basisprojekten - u.a. auch im psychosozialen Bereich - Erfahrung und Kompetenzen gesammelt. Nicht nur in Rojava - sondern überall auf der Welt in langjährigen Kriegsgebieten - ist die Abwanderung von ausgebildetem medizinischem Personal, insbesondere von Ärzten und Ärztinnen, ein grosses Problem. Ärzte wie Dr. Hasin sind eine Seltenheit. Für die Flüchtlinge aus Afrin steht gerade eine einzige 


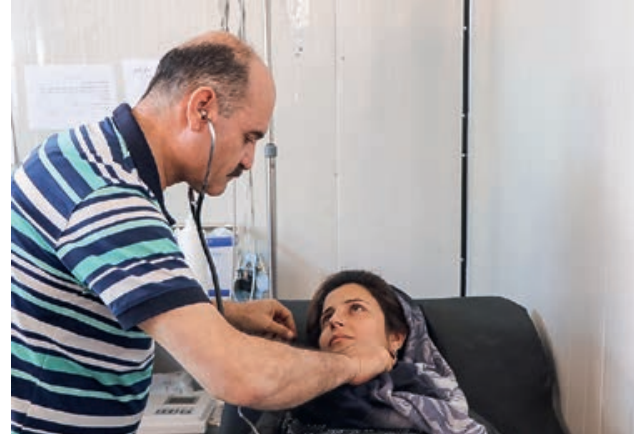

Dr. Hasin untersucht eine Patientin.

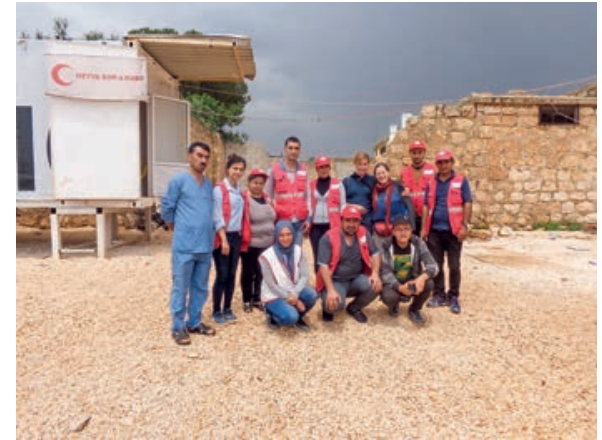

Ein Gesundheitsposten von Heyva Sor a kurd.

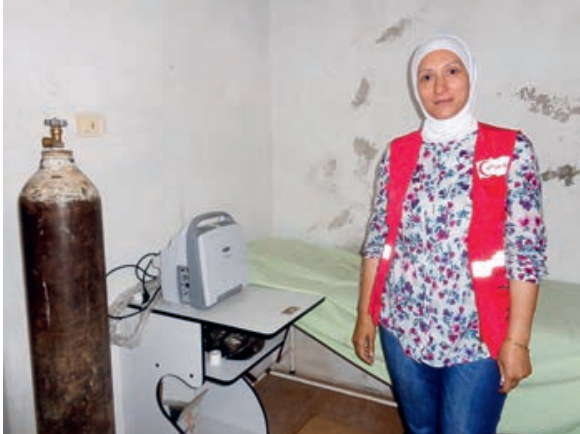

Die Leiterin von Heyva Sor a kurd in Shehba.
Gynäkologin zur Verfügung. Wir diskutieren mit unserer Partnerorganisation deshalb auch die Ausbildung von Gesundheitspromotorinnen nach dem Vorbild von Zentralamerika. Denn inzwischen geniesst medico international schweiz das Vertrauen diverser kurdischer Organisationen aus dem Gesundheitsbereich, aber auch kurdischer Frauenorganisationen.

\section{Wie viele Flüchtlinge aus Afrin leben in der Region Shehba, wie viele in den Zeltlagern? Welche lokalen Organisationen haben diese aufgestellt? Wie war die Stimmung?}

Maja Hess: Schätzungen zum Zeitpunkt unserer Reise sprachen von etwa 110000 Flüchtlingen, sogenannt intern Vertriebenen (IDPs = internally displaced persons). Shehba war noch bis 2016 vom IS besetzt und umkämpft gewesen. Die Menschen aus Afrin entminten leerstehende Dörfer und bauten mit Unterstützung der lokalen Selbstverwaltung und ihrer eigenen kommunalen Strukturen innerhalb von etwa zwei

\section{Niemand bat uns ausschliesslich um finanzielle oder andere materielle Unterstützung.}

Wochen Zeltlager auf. In den drei von mir besuchten Lagern lebten dazumal ca. 6500 Menschen. In jedem Lager, in jedem grösseren Dorf wurde ein Gesundheitsposten des Heyva Sor a kurd (Kurdischer Roter Halbmond, eine lokale in Rojava ansässige medizinische Organisation) eingerichtet. Niemand bat uns ausschliesslich um finanzielle oder andere materielle Unterstützung. Aber alle Flüchtlinge konfrontierten uns immer wieder mit der Frage: Warum hat die Welt nichts gemacht, als das türkische Militär Afrin angriff? Warum hat die UNO geschwiegen, das Rote Kreuz sich zurückgezogen, warum Europa uns vergessen? Die Antwort fiel uns schwer.

\section{Kann medico international schweiz unter den} schwierigen logistischen Umständen in den kurdischen Regionen der Türkei und Syriens überhaupt Projekte realisieren? Welche plant ihr nun in Shehba für die Afrinflüchtlinge? Wer ist dort eure Kontaktinstitution vor Ort?

Maja Hess: Die Umsetzung all unserer «kurdischen» Projekte ist sehr schwierig, kompliziert und politisch heikel. Insbesondere in den kurdischen Gebieten in der Türkei begegnen wir seit dem sogenannten Gülenputsch (Juli 2016) grossen Schwierigkeiten. Unser erster Projektpartner, eine Ärzteorganisation, musste ihre Website schliessen. Dieser Organisation nahestehende Chirurgen hatten während des Kriegs in Kobanê zusammen mit syrischen Ärzten verletzte Zivilisten wie auch verletzte Kämpferinnen und Kämpfer medizinisch behandelt (siehe unser Bericht in der $S A ̈ Z$, 2015;96). Dies hatten sie unter unglaublich prekären Bedingungen getan, zuletzt in einem Keller, nachdem das kobanische Krankenhaus vom IS in die Luft gesprengt worden war. Nach der Befreiung von Kobanê lieferten wir chirurgische und andere medizinische Geräte für den Wiederaufbau eines Spitals in Kobanê. Auch diese Geräte wurden zum Teil in einer Nachtund-Nebel-Aktion über die grüne Grenze gebracht. Inzwischen ist dieser Weg undenkbar: Mit einer $900 \mathrm{~km}$ langen Mauer zwischen der Türkei und Nordsyrien und regelmässigen Grenzschliessungen durch die Barzani-Regierung Nordiraks wurde Rojava völlig isoliert. Aber nicht nur die Lieferung von Material, auch der Geldtransfer ist ein Hürdenlauf. Und auch die Sprachbarriere macht uns zu schaffen. Aktuell arbeiten wir mit Heyva Sor a kurd zusammen. Mit Kreativität und Hartnäckigkeit versucht diese Organisation, Wege zu finden, um die Hindernisse zwischen Rojava und den durch die syrische Regierung kontrollierten Gebieten zu überwinden, um u.a. medizinisches Material zu beschaffen und zu den Flüchtlingen zu 
transportieren. Unser Schwerpunkt ist die allgemeine Hygiene und der Mitaufbau einer minimalsten medizinischen Grundversorgung für die Flüchtlinge aus Afrin: Hygieneartikel, insbesondere für Frauen und Kinder, und pädiatrische Medikamente sowie Arzneimittel für chronische Erkrankungen wie Diabetes, Hypertonie, chronische Nierenerkrankungen etc., alles Produkte von der WHO-Liste essentieller Medikamente.

Der grösste Teil der 2014 während des Kriegs aus der Stadt Kobanê geflüchteten Zivilbevölkerung kehrte nach der Befreiung vom IS durch kurdische Volksverteidigungskräfte (mit Unterstützung der USA) wieder zurück. Wie ist die aktuelle medizinische Versorgung? Therese Vögeli: Über die Hälfte der Geflüchteten ist zurückgekehrt. Heute zählt die Stadt Kobanê ungefähr 100000 Einwohner. Die medizinische Versorgung wird von drei Krankenhäusern, fünf Arztpraxen und 12 Gesundheitsposten auf dem Land sichergestellt. Die

\section{Über die Hälfte der Geflüchteten ist in die Stadt Kobanê zurückgekehrt.}

Gesundheitsposten verfügen über kleine Apotheken und Gesundheitshelfer, aber keine Ärzte. Was allerdings in ganz Rojava nach wie vor nicht behandelt werden kann, sind Krebserkrankungen. Wir konnten zwei Spitäler besuchen, darunter das Frauenspital mit 24 Betten, das vor etwa $1 \frac{1}{2}$ Jahren eröffnet wurde. Im Eingangsbereich des Spitals fielen uns Informationsplakate auf: Mit Bildern wird vor in den Trümmern versteckten Minen gewarnt, die Bauschutt zum Verwechseln ähnlich sehen. Dies machte uns auf verstörende Weise deutlich, wie allgegenwärtig und «normal» die Gräuel des Krieges im Alltag der Menschen hier sind. Übrigens: In Afrin sieht die Lage komplizierter aus. Die Flüchtlinge möchten auch zurück nach Afrin. Dort haben sie - oft nach Jahren der Vertreibung

\section{Mit Bildern wird vor in den Trümmern ver-} steckten Minen gewarnt, die Bauschutt zum Verwechseln ähnlich sehen.

und Flucht - ihr Leben aufgebaut. Wenn es nicht bald gelingt, eine Lösung zu finden, werden Tausende von ihnen versuchen, nach Europa zu gelangen, und irgendwo stranden. Damit die Bemühungen um eine politische Lösung dieser Situation nicht aufgegeben werden, ist es wichtig, die aktuelle provisorische Situation einigermassen erträglich zu machen. Dazu gehört nebst der Basisversorgung mit Lebensmitteln, Hygieneartikeln usw. auch eine angemessene Gesundheitsversorgung. Dazu trägt der Kurdische Rote Halbmond in Rojava mit der Versorgung der Flüchtlinge in Dörfern und Lagern wesentlich bei. Gerade laufen von medico finanzierte medizinische Hilfslieferungen in die Lager. Die politischen und sozialen Organisationen sind sehr bemüht, mit den Flüchtlingen eine Lösung zu finden, damit nicht noch mehr Menschen ihrer Wurzeln und ihrer Identität beraubt werden.

Wer die vorgestellten Gesundheitsprojekte mit einer Spende unterstützen möchte, kann dies tun via Postcheckkonto 80-7869-1, medico international schweiz, Vermerk «Kurdische Gesundheitsprojekte».

\section{Bildnachweis}

Fotos: medico international schweiz

Kartenausschnitt: By AzadG99 (Own work) [CC BY-SA 4.0 (http:// creativecommons.org/licenses/by-sa/4.0)], via Wikimedia Commons. Durch die Autorinnen aktualisiert gemäss Situation Frühling 2018.

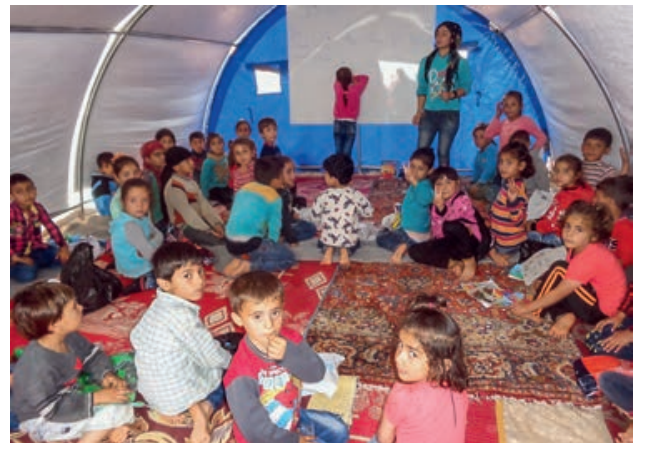

Schulunterricht im Flüchtlingscamp.

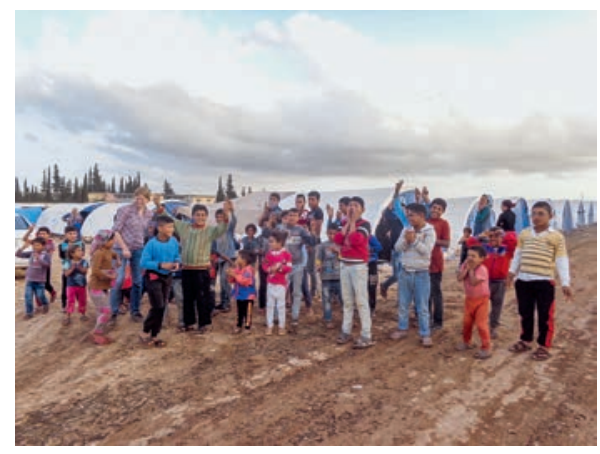

Fotoshooting - eine willkommene Abwechslung für die Kinder im Camp.

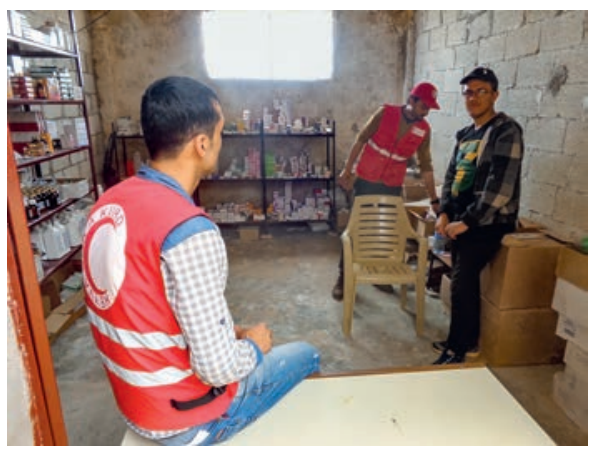

Ein Blick in die Apotheke. 